\title{
Stability of the solutions of nonlinear third order differential equations with multiple deviating arguments
}

\author{
Moussadek Remili \\ Department of Mathematics, \\ University of Oran 1 \\ Ahmed Benbella, Algeria \\ email: remilimous@gmail.com
}

\author{
Lynda Damerdji Oudjedi \\ Department of Mathematics, \\ University of Oran 1 \\ Ahmed Benbella, Algeria \\ email: oudjedi@yahoo.fr
}

\begin{abstract}
In this paper, with use of Lyapunov functional, we investigate asymptotic stability of solutions of some nonlinear differential equations of third order with delay. Our results include and improve some wellknown results in the literature.
\end{abstract}

\section{Introduction}

The investigation of qualitative behavior of solutions such as stability, convergence, boundedness, asymptotic behavior to mention few, are very important problems in the theory and applications of differential equations. For instance, in applied sciences some practical problems concerning mechanics, engineering technique fields, economy, control theory, physical sciences and so on are associated with third, fourth and higher order nonlinear differential equations. In recent years, there has been increasing interest in obtaining sufficient conditions for the asymptotic stability and boundedness of solutions of the nonlinear third order differential equations. Many results relative to the stability, boundedness of solutions of third order differential equations with delays or without

2010 Mathematics Subject Classification: 34C11, 34D20

Key words and phrases: stability, Lyapunov functional, delay differential equations, thirdorder differential equations 
delays have been obtained. We refer the reader to the papers (Burton $[1,2]$, Swick [10] and Yoshizawa [16] and references therein) to discuss the qualitative properties of various form of nonlinear differential equations without delay.

The Lyapunov second method had also been found useful and applicable to study the qualitative properties of the equation with delay. Many interesting results, on the qualitative behavior of solutions of the third order differential equations have been obtained by Omeike [4, 5], Remili and Oudjedi [7], Sadek $[8,9]$, Tunç $[11,12,13,14]$ and Zhu [17] and references therein.

In 2009, the author [5] adapted [10] and used a suitable Lyapunov function to establish criteria which guarantee asymptotic stability of solution of nonautonomous delay differential equation of the third order that is bounded together with its derivatives on the real line, and boundedness under explicit conditions on the nonlinear terms of the equation

$$
x^{\prime \prime \prime}+a(t) x^{\prime \prime}+b(t) g\left(x^{\prime}\right)+c(t) h(x(t-r))=p(t) .
$$

Recently, in 2013 Tunç and Gözen [15] considered the non autonomous differential equation of the third order with multiple deviating arguments:

$$
x^{\prime \prime \prime}+a(t) x^{\prime \prime}+n b(t) g\left(x^{\prime}\right)+c(t) \sum_{i=1}^{n} h_{i}(x(t-r))=p(t) .
$$

He discussed the stability and boundedness of solutions of this equation.

Our aim in this paper, by using Lyapunov second method is to study the asymptotic stability of third-order nonlinear differential equation with multiple deviating arguments

$$
\left[\psi\left(x^{\prime}(t)\right) x^{\prime}(t)\right]^{\prime \prime}+a(t) x^{\prime \prime}(t)+n b(t) g\left(x^{\prime}(t)\right)+c(t) \sum_{i=1}^{n} h_{i}\left(x\left(t-r_{i}\right)\right)=0,
$$

and the boundedness of solutions of the equation

$$
\left[\psi\left(x^{\prime}(t)\right) x^{\prime}(t)\right]^{\prime \prime}+a(t) x^{\prime \prime}(t)+n b(t) g\left(x^{\prime}(t)\right)+c(t) \sum_{i=1}^{n} h_{i}\left(x\left(t-r_{i}\right)\right)=q(t),
$$

where $r_{i}$ are certain positive constants. It is supposed that the derivatives, $a^{\prime}(t), b^{\prime}(t), c^{\prime}(t), \psi^{\prime}(y)=\frac{d \psi}{d y}$, and $h_{i}^{\prime}(x)=\frac{d h_{i}}{d x}$, exist and are continuous.

In this work, we want to adopt the approach in Omeike [5] and Tunç [15] to extend the result in Swick [10] to the equation (1) and give sufficient criteria 
which guarantee the existence of uniform asymptotic stability of the solution with their derivatives on the real line. Obviously, the equations discussed in [5] and [15], are particular cases of our equation (2). Here, by this work, we improve the boundedness result obtained in $[5,15]$.

\section{Preliminaries}

First, we will give some basic definitions and important stability criteria for the general non-autonomous delay differential system. Consider the general non-autonomous delay differential system

$$
x^{\prime}=f\left(t, x_{t}\right), \quad x_{t}(\theta)=x(t+\theta), \quad-r \leq \theta \leq 0, \quad t \geq 0,
$$

where $f: I \times C_{H} \rightarrow \mathbb{R}^{n}$ is a continuous mapping, $f(t, 0)=0, C_{H}:=\{\phi \in$ $\left.\mathrm{C}\left([-\mathrm{r}, 0], \mathbb{R}^{\mathfrak{n}}\right):\|\phi\| \leq \mathrm{H}\right\}$, and for $\mathrm{H}_{1}<\mathrm{H}$, there exists $\mathrm{L}\left(\mathrm{H}_{1}\right)>0$, with $|\mathrm{f}(\mathrm{t}, \phi)|<\mathrm{L}\left(\mathrm{H}_{1}\right)$ when $\|\phi\|<\mathrm{H}_{1}$.

Definition 1 [2] An element $\psi \in \mathrm{C}$ is in the $\omega$ - limit set of $\phi$, say $\Omega(\phi)$, if $\mathrm{x}(\mathrm{t}, 0, \phi)$ is defined on $[0,+\infty)$ and there is a sequence $\left\{\mathrm{t}_{\mathrm{n}}\right\}, \mathrm{t}_{\mathrm{n}} \rightarrow \infty$, as $\mathrm{n} \rightarrow \infty$, with $\left\|\mathrm{x}_{\mathrm{t}_{\mathrm{n}}}(\phi)-\psi\right\| \rightarrow 0$ as $\mathrm{n} \rightarrow \infty$ where $\mathrm{x}_{\mathrm{t}_{\mathrm{n}}}(\phi)=\mathrm{x}\left(\mathrm{t}_{\mathrm{n}}+\theta, 0, \phi\right)$ for $-r \leq \theta \leq 0$.

Definition 2 [2] A set $\mathrm{Q} \subset \mathrm{C}_{\mathrm{H}}$ is an invariant set if for any $\phi \in \mathrm{Q}$, the solution of (3), $x(\mathrm{t}, 0, \phi)$, is defined on $[0, \infty)$ and $\mathrm{x}_{\mathrm{t}}(\phi) \in \mathrm{Q}$ for $\mathrm{t} \in[0, \infty)$.

Lemma 1 [1] If $\phi \in \mathrm{C}_{\mathrm{H}}$ is such that the solution $\mathrm{x}_{\mathrm{t}}(\phi)$ of (3) with $\mathrm{x}_{0}(\phi)=\phi$ is defined on $[0, \infty)$ and $\left\|\mathrm{x}_{\mathrm{t}}(\phi)\right\| \leq \mathrm{H}_{1}<\mathrm{H}$ for $\mathrm{t} \in[0, \infty)$, then $\Omega(\phi)$ is a non-empty, compact, invariant set and

$$
\operatorname{dist}\left(x_{\mathrm{t}}(\phi), \Omega(\phi)\right) \rightarrow 0 \text { as } \mathrm{t} \rightarrow \infty .
$$

Lemma 2 [1] let $\mathrm{V}(\mathrm{t}, \phi): \mathrm{I} \times \mathrm{C}_{\mathrm{H}} \rightarrow \mathbb{R}$ be a continuous functional satisfying a local Lipschitz condition. $\mathrm{V}(\mathrm{t}, \mathrm{O})=\mathrm{0}$, and such that:

(i) $\mathrm{W}_{1}(|\phi(0)|) \leq \mathrm{V}(\mathrm{t}, \phi) \leq \mathrm{W}_{2}(|\phi(0)|)+\mathrm{W}_{3}\left(\|\phi\|_{2}\right)$ where $\|\phi\|_{2}=\left(\int_{t-r}^{t}\|\phi(s)\|^{2} \mathrm{ds}\right)^{\frac{1}{2}}$.

(ii) $\dot{\mathrm{V}}_{(3)}(\mathrm{t}, \phi) \leq-\mathrm{W}_{4}(|\phi(0)|)$, where, $\mathrm{W}_{\mathrm{i}}(\mathrm{i}=1,2,3,4)$ are wedges. Then the zero solution of (3) is uniformly asymptotically stable. 


\section{Assumptions and main results}

The following assumptions will be needed throughout the paper. Let $a_{0}, b_{0}, c_{0}$, $\mathrm{d}, \mathrm{m}, \mathrm{d}_{0}, \mathrm{~d}_{1}, \mathrm{~A}, \mathrm{~B}, \mathrm{C}, \mathrm{L}, \mathrm{M}$, and $\varepsilon, \delta_{i}, \rho_{i}$ be an arbitrary but fixed positives numbers and suppose that $a(t), b(t), c(t) \in C^{1}\left(\mathbb{R}_{+}\right), h \in C^{1}(\mathbb{R}), g \in C(\mathbb{R})$ and let $\psi$ be a twice continuously differential function on IR, such that the following assumptions are satisfied:

i) $\quad 0<\mathrm{a}_{0} \leq \mathrm{a}(\mathrm{t}) \leq \mathrm{A} ; 0<\mathrm{b}_{0} \leq \mathrm{b}(\mathrm{t}) \leq \mathrm{B} ; 0<\mathrm{c}_{0} \leq \mathrm{c}(\mathrm{t}) \leq \mathrm{C}$.

ii) $\quad c(t) \leq b(t), \quad b^{\prime}(t) \leq c^{\prime}(t) \leq 0$ for $t \in[0, \infty)$.

iii) $0<m \leq \psi(u) \leq M ; 0<d_{0} \leq \frac{g(y)}{y} \leq d_{1}$ for $y \neq 0$.

iv) $\quad h_{i}(0)=0, \frac{h_{i}(x)}{x} \geq \delta_{i}>0(x \neq 0)$, and $\left|h_{i}^{\prime}(x)\right| \leq \rho_{i}$ for all $x$.

v) $\frac{M \rho_{i}}{d_{0}}<d<a_{0}$.

vi) $\frac{1}{2} d a^{\prime}(t)-b_{0}\left(d d_{0}-M \sum_{i=1}^{n} \rho_{i}\right) \leq-\varepsilon<0$.

vii) $\int_{-\infty}^{+\infty}\left|\psi^{\prime}(u)\right| d u<\infty$

viii) $\inf _{\mathfrak{u} \in \mathbb{R}} u \Psi^{\prime}(u)=\eta>-m$.

ix) $Q(t)=\int_{0}^{t}|q(s)| d s<\infty$.

For ease of exposition throughout this paper we will adopt the following notation

$$
P(t)=\psi\left(x^{\prime}(t)\right), \quad R(t)=\frac{\psi^{\prime}\left(x^{\prime}(t)\right)}{\psi^{2}\left(x^{\prime}(t)\right)} x^{\prime \prime}(t)
$$

Theorem 1 In addition to conditions (i)-(vii) being satisfied, suppose that the following is also satisfied

$$
\sum_{i=1}^{n} r_{i}<\min \left\{\alpha_{i}, \quad \beta_{i}\right\}
$$


where

$$
\alpha_{i}=\frac{2\left(a_{0}-d\right)}{M C \rho_{i}} \text {, and } \beta_{i}=\frac{2 m^{3} \varepsilon}{C \rho_{i} M^{2}\left(d+d m^{2}+m\right)} .
$$

Then every solution of (1) is uniformly asymptotically stable.

Proof. We write the equation (1) as the following equivalent system

$$
\begin{aligned}
x^{\prime}= & \frac{1}{P(t)} y \\
y^{\prime}= & z \\
z^{\prime}= & -\frac{a(t)}{P(t)} z+a(t) R(t) y-n b(t) g\left(\frac{y}{P(t)}\right)-c(t) \sum_{i=1}^{n} h_{i}(x) \\
& +c(t) \sum_{i=1}^{n} \int_{t-r_{i}}^{t} \frac{y(s)}{P(t)} h_{i}^{\prime}(x(s)) d s .
\end{aligned}
$$

Note that the continuity of the functions $a(t), b(t), c(t), q(t)$ on $[0,+\infty[$, and $\psi\left(x^{\prime}\right), g\left(x^{\prime}\right), h_{i}(x)$ in their respective arguments on $\mathbb{R}$ with $h(0)=g(0)=0$, guarantee the existence of the solution of (4) (see [3]). It is assumed that the right hand side of the system (4) satisfies a Lipschitz condition in $x(t), \chi^{\prime}(t), \chi^{\prime \prime}(t)$ and $x\left(t-r_{i}\right)$. This assumption guarantees the uniqueness of solutions of (4) (see [3], pp.15).

We shall use as a tool to prove our main results a Lyapunov function $\mathrm{U}=$ $\mathrm{u}\left(\mathrm{t}, \mathrm{x}_{\mathrm{t}}, \mathrm{y}_{\mathrm{t}}, z_{\mathrm{t}}\right)$ defined by

$$
\mathrm{u}\left(\mathrm{t}, \mathrm{x}_{\mathrm{t}}, \mathrm{y}_{\mathrm{t}}, z_{\mathrm{t}}\right)=\exp \left(-\frac{\gamma(\mathrm{t})}{\mu}\right) \mathrm{v}\left(\mathrm{t}, \mathrm{x}_{\mathrm{t}}, \mathrm{y}_{\mathrm{t}}, z_{\mathrm{t}}\right)=\exp \left(-\frac{\gamma(\mathrm{t})}{\mu}\right) \mathrm{v},
$$

where

$$
\gamma(\mathrm{t})=\int_{0}^{t}|\mathrm{R}(\mathrm{s})| \mathrm{ds},
$$

and

$$
\begin{aligned}
V= & d c(t) H(x)+c(t) y \sum_{i=1}^{n} h_{i}(x)+n b(t) P(t) G\left(\frac{y}{P(t)}\right)+\frac{1}{2} z^{2} \\
& +\frac{d}{P(t)} y z+\frac{d a(t)}{2 P^{2}(t)} y^{2}+\sum_{i=1}^{n} \lambda_{i} \int_{-r_{i}}^{0} \int_{t+s}^{t} y^{2}(\xi) d \xi d s,
\end{aligned}
$$

where $H(x)=\sum_{i=1}^{n} \int_{0}^{x} h_{i}(u) d u$ and $G(y)=\int_{0}^{y} g(u) d u$. $\mu$ and $\lambda_{i}$ are certain positive constants, which will be specified later in the proof. From the definition 
of $\mathrm{V}$ in (6), we observe that the above Lyapunov functional can be rewritten as follows

$$
V=V_{1}+V_{2}+\sum_{i=1}^{n} \lambda_{i} \int_{-r_{i}}^{0} \int_{t+s}^{t} y^{2}(\xi) d \xi d s,
$$

with

$$
V_{1}=d c(t) H(x)+c(t) y \sum_{i=1}^{n} h_{i}(x)+n b(t) P(t) G\left(\frac{y}{P(t)}\right)
$$

and

$$
v_{2}=\frac{1}{2} z^{2}+\frac{d}{P(t)} y z+\frac{d a(t)}{2 P^{2}(t)} y^{2}
$$

First consider

$$
\begin{aligned}
V_{2} & =\frac{1}{2}\left\{z^{2}+\frac{2 d}{P(t)} y z+\frac{d a(t)}{P^{2}(t)} y^{2}\right\} \\
& =\frac{1}{2}\left(z+\frac{d}{P(t)} y\right)^{2}+\frac{d(a(t)-d)}{2 P^{2}(t)} y^{2} .
\end{aligned}
$$

Using the conditions on $a(t)$ in $(v), \frac{d(a(t)-d)}{2 P^{2}(t)} \geq \frac{d\left(a_{0}-d\right)}{2 P^{2}(t)}>0$, it follows that there exists sufficiently small positive constant $\delta_{2}$ such that

$$
\begin{gathered}
V_{2} \geq \delta_{2}\left(y^{2}+z^{2}\right) . \\
V_{1} \geq d c(t) H(x)+c(t) y \sum_{i=1}^{n} h_{i}(x)+\frac{n d_{0} b(t)}{2 P(t)} y^{2},
\end{gathered}
$$

since $\frac{g(y)}{y} \geq d_{0}>0$ implies that $G\left(\frac{y}{P(t)}\right) \geq \frac{d_{0}}{2 P^{2}(t)} y^{2}$. We wish to arrange $\mathrm{V}_{1}$, and using the assumptions (i)-(v), we get,

$$
\begin{aligned}
V_{1} \geq & d c(t) H(x)+\frac{d_{0} b(t)}{2 P(t)} \sum_{i=1}^{n}\left\{y+\frac{c(t) h_{i}(x) P(t)}{d_{0} b(t)}\right\}^{2} \\
& -\sum_{i=1}^{n} \frac{c^{2}(t) P(t) h_{i}^{2}(x)}{2 d_{0} b(t)} \\
\geq & d c(t) \sum_{i=1}^{n} \int_{0}^{x}\left(1-\frac{c(t) P(t) h_{i}^{\prime}(u)}{d_{0} b(t)}\right) h_{i}(u) d u \\
\geq & d c(t) \sum_{i=1}^{n} \int_{0}^{x}\left(1-\frac{M \rho_{i}}{d d_{0}}\right) h_{i}(u) d u
\end{aligned}
$$




$$
\begin{aligned}
& \geq \operatorname{dc}(t) \sum_{i=1}^{n} \int_{0}^{x}\left(1-\frac{M \rho_{i}}{d d_{0}}\right) \frac{h_{i}(u)}{u} u d u \\
& \geq \operatorname{dc}(t) \sum_{i=1}^{n} \int_{0}^{x}\left(1-\frac{M \rho_{i}}{d d_{0}}\right) \delta_{i} u d u \\
& \geq \frac{d c(t)}{2} \sum_{i=1}^{n}\left(1-\frac{M \rho_{i}}{d d_{0}}\right) \delta_{i} x^{2},
\end{aligned}
$$

so that

$$
V_{1} \geq \frac{\delta_{3}}{2} \chi^{2}
$$

where $\delta_{3}=d c_{0} \sum_{i=1}^{n} \delta_{i}\left(1-\frac{M \rho_{i}}{d d_{0}}\right)>d c_{0} \sum_{i=1}^{n} \delta_{i}\left(1-\frac{d}{d}\right)=0$. From (8),

and (6), It is easy to check that

$$
V \geq \delta_{2} y^{2}+\delta_{2} z^{2}+\frac{\delta_{3}}{2} x^{2}+\sum_{i=1}^{n} \lambda_{i} \int_{-r_{i}}^{0} \int_{t+s}^{t} y^{2}(\xi) d \xi d s .
$$

Subject to the conditions of Theorem $1, \mathrm{~V}(0,0,0)=0$ and there exists sufficiently small positive constant $k$ such that

$$
\mathrm{V} \geq \mathrm{k}\left(\mathrm{x}^{2}+\mathrm{y}^{2}+z^{2}\right)
$$

since the integral $\int_{t+s}^{t} y^{2}(\xi) d \xi$ is positive, where $k=\min \left(\delta_{2}, \frac{\delta_{3}}{2}\right)$.

Assumptions (iii) and (vii) imply the following:

$$
\begin{aligned}
\gamma(\mathrm{t}) & =\int_{0}^{\mathrm{t}}|\mathrm{R}(\mathrm{s})| \mathrm{ds} \\
& \leq \int_{\alpha_{1}(\mathrm{t})}^{\alpha_{2}(\mathrm{t})} \frac{\left|\psi^{\prime}(\tau)\right|}{\psi^{2}(\tau)} \mathrm{d} \tau \\
& \leq \frac{1}{\mathrm{~m}^{2}} \int_{-\infty}^{+\infty}\left|\psi^{\prime}(\tau)\right| \mathrm{d} \tau \leq \mathrm{N}<\infty,
\end{aligned}
$$

where $\alpha_{1}(t)=\min \left\{x^{\prime}(0), x^{\prime}(t)\right\}$, and $\alpha_{2}(t)=\max \left\{x^{\prime}(0), x^{\prime}(t)\right\}$. Now, we can deduce that there exists a continuous function $W_{1}$ with $W_{1}(|\Phi(0)|) \geq 0$ such that $\mathrm{W}_{1}(|\Phi(0)|) \leq \mathrm{U}(\mathrm{t}, \Phi)$.

The existence of a continuous function $W_{2}(\|\phi\|)$ which satisfies the inequality $\mathrm{U}(\mathrm{t}, \phi) \leq \mathrm{W}_{2}(\|\phi\|)$, is easily verified. 
Now, let $(x, y, z)=(x(t), y(t), z(t))$ be any solution of differential system (4).

Differentiating the function $\mathrm{V}$, defined in (6), along system (4) with respect to the independent variable $t$, we have

$$
\begin{aligned}
& \frac{d}{d t} V=d c^{\prime}(t) H(x)+c^{\prime}(t) y \sum_{i=1}^{n} h_{i}(x)+n b^{\prime}(t) P(t) G\left(\frac{y}{P(t)}\right)+\frac{d-a(t)}{P(t)} z^{2} \\
& +R(t)\left[(a(t)-d) z y-n b(t) P(t)\left(g\left(\frac{y}{P(t)}\right) y-P(t) G\left(\frac{y}{P(t)}\right)\right)\right]+\sum_{i=1}^{n} \lambda_{i} r_{i} y^{2} \\
& +\left[\frac{d a^{\prime}(t)+2 c(t) P(t) \sum_{i=1}^{n} h_{i}^{\prime}(x)}{2 P^{2}(t)} y^{2}-n d b(t) \frac{y}{P(t)} g\left(\frac{y}{P(t)}\right)\right] \\
& +c(t)\left(z+\frac{d y}{P(t)}\right) \sum_{i=1}^{n} \int_{t-r_{i}}^{t} \frac{y(s)}{P(s)} h_{i}^{\prime}(x(s)) d s-\sum_{i=1}^{n} \lambda_{i} \int_{t-r_{i}}^{t} y^{2}(\xi) d \xi .
\end{aligned}
$$

Consequently by the hypothesis (i)-(vi), it follows that

$$
\begin{aligned}
\frac{d}{d t} V \leq & d c^{\prime}(t) H(x)+c^{\prime}(t) y \sum_{i=1}^{n} h_{i}(x)+\frac{n d_{0} b^{\prime}(t)}{2 P(t)} y^{2}-\left(\frac{\varepsilon}{M^{2}}-\sum_{i=1}^{n} \lambda_{i} r_{i}\right) y^{2} \\
& +|R(t)|\left[(A-d)|z y|+\frac{3}{2} n B d_{1} y^{2}\right]-\frac{1}{M}\left(a_{0}-d\right) z^{2} \\
& +c(t)\left(z+\frac{d y}{P(t)}\right) \sum_{i=1}^{n} \int_{t-r_{i}}^{t} \frac{y(s)}{P(s)} h_{i}^{\prime}(x(s)) d s-\sum_{i=1}^{n} \lambda_{i} \int_{t-r_{i}}^{t} y^{2}(\xi) d \xi
\end{aligned}
$$

We claim that

$$
\theta(t, x, y)=d c^{\prime}(t) H(x)+c^{\prime}(t) y \sum_{i=1}^{n} h_{i}(x)+\frac{n d_{0} b^{\prime}(t)}{2 P(t)} y^{2} \leq 0
$$

for all $x, y$ and $t \geq 0$. First suppose that $c^{\prime}(t)=0$, then

$$
\theta(t, x, y)=\frac{n d_{0} b^{\prime}(t)}{2 P(t)} y^{2} \leq 0
$$

Finally, suppose that $c^{\prime}(t)<0$, the quantity in the brackets above can be 
written as,

$$
\begin{aligned}
\theta(t, x, y)= & d c^{\prime}(t)\left[H(x)+\frac{1}{d} y \sum_{i=1}^{n} h_{i}(x)+\frac{n d_{0} b^{\prime}(t)}{2 d c^{\prime}(t) P(t)} y^{2}\right] \\
= & d c^{\prime}(t)\left[H(x)+\frac{d_{0} b^{\prime}(t)}{2 d c^{\prime}(t) P(t)} \sum_{i=1}^{n}\left\{y+\frac{c^{\prime}(t) P(t) h_{i}(x)}{d_{0} b^{\prime}(t)}\right\}^{2}\right] \\
& -d c^{\prime}(t)\left[\sum_{i=1}^{n} \frac{c^{\prime}(t) P(t) h_{i}^{2}(x)}{2 d d_{0} b^{\prime}(t)}\right],
\end{aligned}
$$

moreover, assumption (ii) implies $\frac{\mathrm{c}^{\prime}(\mathrm{t})}{\mathrm{b}^{\prime}(\mathrm{t})} \leq 1$, thus

$$
\begin{aligned}
\theta(t, x, y) & \leq d c^{\prime}(t) \sum_{i=1}^{n} \int_{0}^{x}\left(1-\frac{P(t) h_{i}^{\prime}(u)}{d d_{0}}\right) h_{i}(u) d u \\
& \leq d c^{\prime}(t) \sum_{i=1}^{n} \int_{0}^{x}\left(1-\frac{M \rho_{i}}{d d_{0}}\right) h_{i}(u) d u \\
& \leq c^{\prime}(t) \frac{\delta_{3}}{2 c_{0}} x^{2} \leq 0 .
\end{aligned}
$$

Hence, on combining the two cases, we have $\theta(t, x, y) \leq 0$ for all $t \geq 0, x$ and $y$. Utilizing the assumptions of theorem and Schwartz inequality $|u v| \leq \frac{1}{2}\left(u^{2}+v^{2}\right)$, the following inequalities are obtained

$$
\begin{aligned}
\frac{d c(t)}{P(t)} y \sum_{i=1}^{n} \int_{t-r_{i}}^{t} \frac{y(s)}{P(s)} h_{i}^{\prime}(x(s)) d s & \leq \sum_{i=1}^{n} \frac{d C \rho_{i} r_{i}}{2 m} y^{2}+\frac{C d}{2 m^{3}} \sum_{i=1}^{n} \int_{t-r_{i}}^{t} \rho_{i} y^{2}(\xi) d \xi \\
& \leq \sum_{i=1}^{n} \frac{d C \rho_{i} r_{i}}{2 m} y^{2}+\frac{C d \rho_{i}}{2 m^{3}} \sum_{i=1}^{n} \int_{t-r_{i}}^{t} y^{2}(\xi) d \xi \\
c(t) z \sum_{i=1}^{n} \int_{t-r_{i}}^{t} \frac{y(s)}{P(s)} h_{i}^{\prime}(x(s)) d s & \leq \sum_{i=1}^{n} \frac{C \rho_{i} r_{i}}{2} z^{2}+\frac{C}{2 m^{2}} \sum_{i=1}^{n} \int_{t-r_{i}}^{t} \rho_{i} y^{2}(\xi) d \xi \\
& \leq \sum_{i=1}^{n} \frac{C \rho_{i} r_{i}}{2} z^{2}+\frac{C \rho_{i}}{2 m^{2}} \sum_{i=1}^{n} \int_{t-r_{i}}^{t} y^{2}(\xi) d \xi
\end{aligned}
$$


and

$$
\begin{aligned}
W_{1} & =|R(t)|\left[(A-d)|z y|+\frac{3}{2} n B d_{1} y^{2}\right] \\
& \leq|R(t)|\left[\frac{A-d}{2} z^{2}+\frac{A-d+3 n B d_{1}}{2} y^{2}\right] \\
& \leq k_{1}|R(t)|\left(y^{2}+z^{2}\right),
\end{aligned}
$$

where $k_{1}=\frac{A-d+3 n B d_{1}}{2}$. These estimates imply that

$$
\begin{aligned}
\frac{d}{d t} V \leq & -\left[\frac{\varepsilon}{M^{2}}-\sum_{i=1}^{n}\left(\lambda_{i}+\frac{d C \rho_{i}}{2 m}\right) r_{i}\right] y^{2} \\
& -\left[\frac{a_{0}-d}{M}-\sum_{i=1}^{n} \frac{C \rho_{i} r_{i}}{2}\right] z^{2} \\
& +\sum_{i=1}^{n}\left[\frac{C \rho_{i}}{2 m^{2}}\left(1+\frac{d}{m}\right)-\lambda_{i}\right] \int_{t-r_{i}}^{t} y^{2}(\xi) d \xi \\
& +k_{1}|R(t)|\left(y^{2}+z^{2}\right) .
\end{aligned}
$$

If we take $\frac{C \rho_{i}}{2 m^{2}}\left(1+\frac{d}{m}\right)=\lambda_{i}$, the last inequality becomes

$$
\begin{aligned}
\frac{d}{d t} V \leq & -\left[\frac{\varepsilon}{M^{2}}-\sum_{i=1}^{n} \frac{C \rho_{i}}{2 m}\left(d+\frac{1}{m}+\frac{d}{m^{2}}\right) r_{i}\right] y^{2} \\
& -\left[\frac{a_{0}-d}{M}-\sum_{i=1}^{n} \frac{C \rho_{i} r_{i}}{2}\right] z^{2}+k_{1}|R(t)|\left(y^{2}+z^{2}\right) .
\end{aligned}
$$

Using (9), (5) and taking $\mu=\frac{k}{k_{1}}$ we obtain:

$$
\begin{aligned}
\frac{d}{d t} \mathrm{U}= & \exp \left(-\frac{k_{1} \gamma(t)}{k}\right)\left(\frac{d}{d t} V-\frac{k_{1}|R(t)|}{k} V\right) \\
\leq & \exp \left(-\frac{k_{1} \gamma(t)}{k}\right)\left[-\left(\frac{\varepsilon}{M^{2}}-\sum_{i=1}^{n} \frac{C \rho_{i} r_{i}}{2 m}\left(d+\frac{1}{m}+\frac{d}{m^{2}}\right)\right) y^{2}\right. \\
& \left.-\left(\frac{a_{0}-d}{M}-\sum_{i=1}^{n} \frac{C \rho_{i} r_{i}}{2}\right) z^{2}\right] .
\end{aligned}
$$


Provided that

$$
\sum_{i=1}^{n} r_{i}<\min \left\{\frac{2\left(a_{0}-d\right)}{M C \rho_{i}}, \frac{2 m^{3} \varepsilon}{C \rho_{i} M^{2}\left(d+d m^{2}+m\right)}\right\} .
$$

The inequality (10) becomes

$$
\frac{d}{d t} u\left(t, x_{t}, y_{t}, z_{t}\right) \leq-\beta \exp \left(-\frac{k_{1} N}{k}\right)\left(y^{2}+z^{2}\right), \text { for some } \beta>0 .
$$

It is clear that the largest invariant set in $Z$ is $Q=\{0\}$, where

$$
Z=\left\{\phi \in C_{H}: \frac{d}{d t} U(\phi)=0\right\} .
$$

Namely, the only solution of system (4) for which $\frac{d}{d t} u\left(t, x_{t}, y_{t}, z_{t}\right)=0$ is the solution $x=y=z=0$. Thus, we conclude that every solution of system (4) is uniformly asymptotically stable. Now from (4) we have

$$
x^{\prime}(t) \Psi\left(x^{\prime}(t)\right)=y(t),
$$

Furthermore, it follows from (iii) that

$$
\frac{|y(t)|}{M} \leq\left|x^{\prime}(t)\right|=\frac{|y(t)|}{\Psi\left(x^{\prime}(t)\right)} \leq \frac{|y(t)|}{m},
$$

which implies that $\lim _{\mathrm{t} \rightarrow \infty} x^{\prime}(\mathrm{t})=0$. Differentiating (11) we obtain

$$
x^{\prime \prime}(\mathrm{t})\left[\Psi\left(x^{\prime}(\mathrm{t})\right)+\Psi^{\prime}\left(x^{\prime}(\mathrm{t})\right) x^{\prime}(\mathrm{t})\right]=z(\mathrm{t})
$$

then $\lim _{t \rightarrow \infty} x^{\prime \prime}(t)=0$ since $\lim _{t \rightarrow \infty} \Psi\left(x^{\prime}(t)\right)+\Psi^{\prime}\left(x^{\prime}(t)\right) x^{\prime}(t)=\Psi(0)$.

Thus, under the above discussion, we conclude that every solution of equation (1) is uniformly asymptotically stable.

For the case $\mathrm{q}(\mathrm{t}) \neq 0$, we consider the equivalent system of (2)

$$
\begin{aligned}
x^{\prime}= & \frac{1}{P(t)} y \\
y^{\prime}= & z \\
z^{\prime}= & -\frac{a(t)}{P(t)} z+a(t) R(t) y-n b(t) g\left(\frac{y}{P(t)}\right)-c(t) \sum_{i=1}^{n} h_{i}(x) \\
& +c(t) \sum_{i=1}^{n} \int_{t-r_{i}}^{t} \frac{y(s)}{P(t)} h_{i}^{\prime}(x(s)) d s+q(t) .
\end{aligned}
$$

The following result is introduced. 
Theorem 2 In addition to the assumptions of Theorem 1, we assume that (viii) and (ix) hold. Then, there exists a finite positive constant $\mathrm{C}$ such that every solution $\mathrm{x}(\mathrm{t})$ of equation (2) defined by the initial functions

$$
x(0)=\phi(t), \quad x^{\prime}(0)=\phi^{\prime}(t), \quad x^{\prime \prime}(0)=\phi^{\prime \prime}(t),
$$

satisfies the inequalities

$$
|x(t)| \leq C, \quad\left|x^{\prime}(t)\right| \leq C, \quad\left|x^{\prime \prime}(t)\right| \leq C \quad \forall t \geq 0,
$$

where $\phi \in \mathrm{C}^{2}([-r, 0], \mathbb{R})$.

Proof. An easy calculation from (13) and (5) yields that

$$
\frac{d}{d t} u_{(11)}=\frac{d}{d t} u_{(4)}+\left(z+\frac{d}{P(t)} y\right) q(t) .
$$

Since $\frac{\mathrm{d}}{\mathrm{dt}} \mathrm{U}_{(4)} \leq 0$, then it follows that

$$
\frac{\mathrm{d}}{\mathrm{dt}} \mathrm{u}_{(11)} \leq\left(|z|+\frac{\mathrm{d}}{\mathrm{P}(\mathrm{t})}|\mathrm{y}|\right)|\mathrm{q}(\mathrm{t})|
$$

Noting that $|x| \leq 1+x^{2}$, which implies that

$$
\begin{aligned}
\left(|z|+\frac{\mathrm{d}}{\mathrm{P}(\mathrm{t})}|\mathrm{y}|\right)|\mathrm{q}(\mathrm{t})| & \leq \mathrm{k}_{2}(|z|+|\mathrm{y}|)|\mathrm{q}(\mathrm{t})| \\
& \leq \mathrm{k}_{2}\left(2+z^{2}+\mathrm{y}^{2}\right)|\mathrm{q}(\mathrm{t})| \\
& \leq \mathrm{k}_{2}\|\mathrm{X}\|^{2}|\mathrm{q}(\mathrm{t})|+2 \mathrm{k}_{2}|\mathrm{q}(\mathrm{t})| \\
& \leq \frac{\mathrm{k}_{2}}{\delta e^{-\frac{N}{\mu}}}|\mathrm{q}(\mathrm{t})| \mathrm{U}+2 \mathrm{k}_{2}|\mathrm{q}(\mathrm{t})|,
\end{aligned}
$$

where $k_{2}=\max \left\{1, \frac{d}{m}\right\}$, recalling that

$$
\delta e^{-\frac{N}{\mu}}\|X\|^{2} \leq u\left(t, x_{t}, y_{t}, z_{t}\right) .
$$

Let $\eta=\max \left\{2 k_{2}, \frac{k_{2}}{\delta e^{-\frac{N}{\mu}}}\right\}$, then

$$
\frac{d}{d t} u_{(11)} \leq \eta|q(t)|+\eta|q(t)| u .
$$


Multiplying each side of this inequality by the integrating factor $e^{-\eta \mathrm{Q}(\mathrm{t})}$, we get

$$
e^{-\eta Q(t)} \frac{d}{d t} U_{(11)} \leq e^{-\eta Q(t)} \eta Q^{\prime}(t)+e^{-\eta Q(t)} \eta Q^{\prime}(t) U .
$$

Integrating each side of this inequality from 0 to $t$, we get, where $X_{0}=$ $(x(0), y(0), z(0))$,

$$
e^{-\eta Q(t)} U-U\left(0, X_{0}\right) \leq 1-e^{-\eta Q(t)} .
$$

Since $\mathrm{Q}(\mathrm{t}) \leq \mathrm{L}$ for all $\mathrm{t}$, we have

$$
\mathrm{u}\left(\mathrm{t}, \mathrm{x}_{\mathrm{t}}, \mathrm{y}_{\mathrm{t}}, z_{\mathrm{t}}\right) \leq \mathrm{u}\left(0, \mathrm{X}_{0}\right) e^{\eta \mathrm{L}}+\left[e^{\eta \mathrm{L}}-1\right] \text { for } \mathrm{t} \geq 0 .
$$

Now, since the right-hand side is a constant, and since $\mathrm{U}\left(\mathrm{t}, \mathrm{x}_{\mathrm{t}}, \mathrm{y}_{\mathrm{t}}, z_{\mathrm{t}}\right) \rightarrow \infty$ as $x^{2}+y^{2}+z^{2} \rightarrow \infty$, it follows that there exists a $D>0$ such that

$$
|x(t)| \leq D,|y(t)| \leq D,|z(t)| \leq D \quad \forall t \geq 0 .
$$

From (11) and (iii) we obtain

$$
\left|x^{\prime}\right|=\left|\frac{y}{\Psi\left(x^{\prime}\right)}\right| \leq \frac{D}{m},
$$

it follows from condition (viii) that

$$
K\left(x^{\prime}\right)=\psi\left(x^{\prime}\right)+x^{\prime} \psi^{\prime}\left(x^{\prime}\right) \geq m+\eta,
$$

but (12) implies

$$
\left|x^{\prime \prime}\right|=\frac{|z|}{K\left(x^{\prime}\right)} \leq \frac{D}{\eta+m}
$$

thus we can deduce

$$
|x(t)| \leq C,\left|x^{\prime}(t)\right| \leq C,\left|x^{\prime \prime}(t)\right| \leq C \quad \forall t \geq 0,
$$

where $C=\sup \left(D, \frac{D}{m}, \frac{D}{\eta+m}\right)$. This completes the proof of theorem.

\section{Example 1}

$$
\begin{aligned}
& \left(\left(\frac{x^{\prime}}{1+x^{\prime 2}}+n(n+1)\right) x^{\prime}\right)^{\prime \prime}+\left(4 n^{2}(n+1)^{2}-\frac{1}{2} e^{-2 t}+\frac{1}{2}\right) x^{\prime \prime} \\
& \quad+n\left(\frac{1}{1+t}+1\right)\left(2 x^{\prime}+\frac{x^{\prime}}{1+x^{\prime 2}}\right) \\
& \quad+\left(\frac{1}{2(1+t)}+\frac{1}{2}\right) \sum_{i=1}^{n}\left[i x\left(t-r_{i}\right)+\frac{i x\left(t-r_{i}\right)}{1+\left|x\left(t-r_{i}\right)\right|}\right]=e^{-t}
\end{aligned}
$$


We can simply verify that

i) $4 n^{2}(n+1)^{2}=a_{0} \leq a(t)=4 n^{2}(n+1)^{2}-\frac{1}{2} e^{-2 t}+\frac{1}{2} \leq 4 n^{2}(n+1)^{2}+\frac{1}{2}, t \geq 0$, $\mathrm{c}_{0}=\frac{1}{2} \leq \mathrm{c}(\mathrm{t})=\frac{1}{2(1+\mathrm{t})}+\frac{1}{2} \leq \mathrm{C}=1=\mathrm{b}_{0} \leq \mathrm{b}(\mathrm{t})=\frac{1}{1+\mathrm{t}}+1 \leq 2, \mathrm{t} \geq 0$,

ii) From (i) we have $\mathrm{b}(\mathrm{t})>\mathrm{c}(\mathrm{t})$ and $\mathrm{b}^{\prime}(\mathrm{t}) \leq \mathrm{c}^{\prime}(\mathrm{t}) \leq 0, \forall \mathrm{t} \geq 0$,

iii) $\psi\left(x^{\prime}\right)=\frac{x^{\prime}}{1+x^{\prime 2}}+n(n+1)$. Now, it is easy to see that

$$
\begin{aligned}
& \inf _{u \in \mathbb{R}} \Psi(u)=-\frac{1}{2}+n(n+1)>m=-1+n(n+1), \\
& \sup _{u \in \mathbb{R}} \Psi(u)=\frac{1}{2}+n(n+1)<M=1+n(n+1), \\
d_{0}= & 2 \leq \frac{g(y)}{y}=2+\frac{1}{1+y^{2}} \leq 3=d_{1} \text { with } y \neq 0 .
\end{aligned}
$$

Also

iv) $\delta_{i}=i \leq \frac{h_{i}(x)}{x}=\left(i+\frac{i}{1+|x|}\right)$ with $x \neq 0$, and $\left|h_{i}^{\prime}(x)\right| \leq \rho_{i}=2 i$,

then $\sum_{i=1}^{n} \rho_{i}=\sum_{i=1}^{n} 2 i=n(n+1)$.

v) For $\mathrm{d}=2 \mathrm{Mn}(\mathrm{n}+1)$ we have

$$
M i=\frac{M \rho_{i}}{d_{0}}<M n<d<a_{0}=4 n^{2}(n+1)^{2},
$$

vi) $a^{\prime}(t)=e^{-2 t} \leq 1$, and

$$
\frac{1}{2} d a^{\prime}(t)-b_{0}\left(d d_{0}-M \sum_{i=1}^{n} \rho_{i}\right) \leq-\frac{3}{2} d+M n(n+1)<0 .
$$

vii) An explicit calculation shows that

$$
\begin{aligned}
\int_{-\infty}^{+\infty}\left|\psi^{\prime}(u)\right| d u=\int_{-\infty}^{+\infty}\left|\frac{u^{2}+1-2}{\left(1+u^{2}\right)^{2}}\right| d u & \leq \int_{-\infty}^{+\infty}\left[\left|\frac{1}{1+u^{2}}\right|+\left|\frac{2}{\left(1+u^{2}\right)^{2}}\right|\right] d u \\
& \leq 2 \pi,
\end{aligned}
$$

viii) $\inf _{\mathfrak{u} \in \mathbb{R}} u \Psi^{\prime}(u)=\eta=-\frac{1}{4}>-n(n+1)+1$, 
ix) $\mathrm{Q}(\mathrm{t})=\int_{0}^{\mathrm{t}} \mathrm{e}^{-\mathrm{s}} \mathrm{ds}<\infty$.

If we take $r_{i}=\frac{2 k}{\pi^{2} i^{2}}$, with $k=\min \left\{\alpha_{n}, \beta_{n}\right\}$. Then

$$
\sum_{i=1}^{i=n} r_{i}<\sum_{i=1}^{\infty} \frac{2 k}{\pi^{2} i^{2}}=k<\min \left\{\alpha_{i}, \beta_{i}\right\} .
$$

All the assumptions (i) through (ix) are satisfied, we can conclude using Theorem 3.2 that every solution of (14) is uniformly bounded.

\section{References}

[1] T. A. Burton, Stability and periodic solutions of ordinary and functional differential equations, Mathematics in Science and Engineering, 178. Academic Press, Inc., Orlando, FL, 1985.

[2] T. A. Burton, Volterra Integral and Differential Equations, Mathematics in Science and Engineering V(202) (2005), 2nd edition.

[3] L. É. Él'sgol'ts, Introduction to the theory of differential equations with deviating arguments, Translated from the Russian by Robert J. McLaughlin Holden-Day, Inc., San Francisco, Calif. London-Amsterdam, 1966.

[4] M. O. Omeike, New results on the stability of solution of some nonautonomous delay differential equations of the third order, Differential Equations and Control Processes, 1 (2010), 18-29.

[5] M. O. Omeike, Stability and boundedness of solutions of some nonautonomous delay differential equation of the third order, An. Stiint. Univ. Al. I. Cuza Iasi. Mat. (N.S.), 55 (1) (2009), 49-58.

[6] M. Remili, D. Beldjerd. On the asymptotic behavior of the solutions of third order delay differential equations, Rend. Circ. Mat. Palermo 63 (2014), 447-455.

[7] M. Remili, L. Damerdji Oudjedi, Stability and Boundedness of the Solutions of Non Autonomous Third Order Differential Equations with Delay, Acta Univ. Palacki. Olomuc., Fac. rer. nat., Mathematica, 53 (2) (2014), 139-147. 
[8] A. I. Sadek, On the Stability of Solutions of Some Non-Autonomous Delay Differential Equations of the Third Order, Asymptot. Anal., 43 (1-2) (2005),1-7.

[9] A. I. Sadek, Stability and Boundedness of a Kind of Third-Order Delay Differential System, Applied Mathematics Letters, 16 (5) (2003), 657-662.

[10] K. Swick, On the boundedness and the stability of solutions of some nonautonomous differential equations of the third order, J. London Math. Soc. 44 (1969), 347-359.

[11] C. Tunç, On asymptotic stability of solutions to third order nonlinear differential equations with retarded argument, Communications in applied analysis, 11 (4) (2007), 515-528.

[12] C. Tunç, On the stability and boundedness of solutions to third order nonlinear differential equations with retarded argument, Nonlinear Dynam. 57 (1-2) (2009), 97-106.

[13] C. Tunç, Some stability and boundedness conditions for non-autonomous differential equations with deviating arguments, E. J. Qualitative Theory of Diff. Equ., 1 (2010), 1-12.

[14] C. Tunç, Stability and boundedness of solutions of nonlinear differential equations of third-order with delay, Journal Differential Equations and Control Processes (Differentsialprimnye Uravneniyai Protsessy Upravleniya), 3 (2007), 1-13.

[15] C. Tunç, M. Gözen, Stability and Uniform Boundedness in Multidelay Functional Differential Equations of Third Order, Hindawi Publishing Corporation Abstract and Applied Analysis. Volume 2013, Article ID 248717, 7 pages.

[16] T. Yoshizawa, Stability theory by Liapunov's second method, The Mathematical Society of Japan, Tokyo, 1966.

[17] Y. F. Zhu, On stability, boundedness and existence of periodic solution of a kind of third order nonlinear delay differential system. Ann. Differential Equations, 8 (2) (1992), 249-259. 